\title{
Assurance qualité des huileries tunisiennes
}

\author{
Ines Gharbi ${ }^{\star}$, Manel Issaoui, Sounira Mehri et Mohammed Hammami \\ Laboratore de Biochimie, LR 12ES 05 Lab-NAFS Nutrition - Aliments Fonctionnels et Santé vasculaire Faculté de Medicine, Université \\ de Monastir, Monastir, Tunisie
}

Reçu le 14 février 2015 - Accepté le 8 mai 2015

\begin{abstract}
Résumé - L'huile d'olive est largement appréciée dans le monde entier pour ses qualités nutritionnelles, ses effets bénéfiques sur la santé et ses caractères organoleptiques. En Tunisie, l'huile d'olive est un produit stratégique d'exportation. Il joue un rôle économique, social et environnemental important en contribuant à la réalisation des objectifs nationaux de sécurité alimentaire, de création d'emploi, d'équilibre de la balance commerciale, de préservation des ressources naturelles et de limitation de l'exode rural. Sur le plan international, il procure à la Tunisie le rang du quatrième producteur et du troisième exportateur mondial et des parts non négligeables sur les principaux marchés d'exportation. L'objectif principal de ce travail est d'étudier les principaux facteurs influençant la qualité des huiles d'olive tunisiennes en mettant l'accent sur l'importance de la mise en place du système HACCP (Hasard analysis of critical control points) au sein des unités de trituration et ceci à travers une enquête sur le terrain et des analyses physicochimiques de 89 échantillons d'huiles d'olives produites dans la région du sahel et du centre Tunisien.
\end{abstract}

Mots clés : Huile d'olives / qualité / HACCP / extraction / huileries tunisiennes

\begin{abstract}
Quality insurance of tunisian olive oils. Olive oil is worldwide appreciated for its nutritional qualities, its beneficial health effects and its organoleptic characteristics. In Tunisia, olive oil is a strategic product for export. It plays an important economic, social and environmental role by contributing to the achievement of national food security objectives, job creation, equilibrium of the commercial balance, preservation of natural resources and limiting the rural exodus. Internationally, it provides for Tunisia the rank of the fourth largest producer and the third largest exporter and significant parts in the principal exportation markets. The main objective of this work is to study the main factors influencing the quality of Tunisian olive oil with emphasis on the importance of the implementation of HACCP (Hazard analysis of critical control points) in olive oil mills and this through a field investigation and physicochemical analyzes of 89 samples of olive oils produced in the region of Sahel and Center of Tunisia.
\end{abstract}

Keywords: Olive oil / quality / HACCP / extraction / Tunisian olive oil mills

\section{Introduction}

Depuis la plus haute antiquité, l'olivier, comme l'huile que l'on en tire, est le symbole sacré de la force, de la lumière et de la vie. En Tunisie, l'huile d'olive est un produit stratégique d'exportation. Il joue un rôle économique, social et environnemental important. En outre, il contribue à la réalisation des objectifs nationaux de sécurité alimentaire, de création d'emploi, d'équilibre de la balance commerciale, de préservation des ressources naturelles et de limitation de l'exode rural. Sur le plan international, il procure à la Tunisie le rang du quatrième producteur et du troisième exportateur mondial et des parts non négligeables sur les principaux marchés d'exportation (Karray et al., 2009). Le potentiel oléicole est localisé pour $30 \%$ au nord, $38 \%$ au centre et $32 \%$ au sud. Il est ré-

^ Correspondance : inesgharbi27@yahoo.fr parti dans des conditions bioclimatiques très variées. Cependant, cette oléiculture est axée sur deux cultivars dominants, le cultivar Chétoui, dans le Nord, et le cultivar Chemlali dans le Centre et le Sud du pays (Hannachi et al., 2007).

L'olivier à huile fait fonctionner un tissu industriel renfermant 1702 huileries (719 classiques, 450 super-presses, 515 chaînes continues et 18 mixtes) ayant une capacité théorique de trituration d'olives de 38463 tonnes par jour. La répartition géographique de ces huileries révèle une grande concentration des unités et de la capacité de trituration des olives dans les régions de Sfax (410), Mahdia (229), Sousse (195), Monastir (187), Médenine (137), Kairouan (115), Sidi Bouzid (85), Nabeul (59), Gafsa (50), Gabes (35), Zaghouan (30), Béja (28) et Mannouba (17) qui représentent 92,7\% des huileries (Karray et al., 2009).

Selon la définition du COI, l'huile d'olive est l'huile provenant uniquement du fruit de l'olivier (Olea europaea L.). 
Elle est commercialisée selon les dénominations et définitions ci-après :

- Les huiles d'olive vierges sont : les huiles obtenues du fruit de l'olivier uniquement par des procédés mécaniques ou d'autres procédés physiques dans des conditions, thermiques notamment, qui n'entraînent pas d'altération de l'huile, et n'ayant subi aucun traitement autre que le lavage, la décantation, la centrifugation et la filtration.

- Les huiles d'olive vierges propres à la consommation en l'état comportent :

- Huile d'olive vierge extra : huile d'olive vierge dont l'acidité libre exprimée en acide oléique est au maximum de $0,8 \mathrm{~g}$ pour $100 \mathrm{~g}$.

- Huile d'olive vierge : huile d'olive vierge dont l'acidité libre exprimée en acide oléique est au maximum de $2 \mathrm{~g}$ pour $100 \mathrm{~g}$.

- Huile d'olive vierge courante : huile d'olive vierge dont l'acidité libre exprimée en acide oléique est au maximum de $3,3 \mathrm{~g}$ pour $100 \mathrm{~g}$.

- Huile d'olive vierge non propre à la consommation en l'état, dénommée huile d'olive vierge lampante, est l'huile d'olive vierge dont l'acidité libre exprimée en acide oléique est supérieure à 3,3 g pour $100 \mathrm{~g}$. Elle est destinée aux industries du raffinage ou à des usages techniques.

La qualité de l'huile d'olive vierge, la seule huile alimentaire pouvant prétendre au qualificatif de «naturelle», est un atout majeur parce qu'elle est intimement liée aux valeurs nutritionnelle, biologique et organoleptique de l'huile. Une moindre qualité des huiles d'olive nuit à leur image de marque qui justifiait jusque là, pour le consommateur, leur prix relativement élevé par rapport aux huiles de graine.

La qualité de l'huile d'olive varie non seulement en fonction de la variété, du sol et des conditions climatiques mais également avec de nombreux facteurs ayant trait au cycle de production, de transformation et de commercialisation des olives et des huiles (Chimi, 2001). Le présent travail décrit l'état actuel de la filière oléicole en Tunisie en rapportant ses points faibles afin de produire une huile d'olive de meilleure qualité et répondant mieux aux exigences du marché international.

\section{Materiels et methodes}

L'approche méthodologique a été envisagée selon trois axes : une enquête sur le terrain, des analyses physicochimiques au laboratoire et une analyse statistique.

L'enquête sur le terrain porte sur:

- la qualité de la matière première produite;

- les aspects technologiques des unités de trituration des olives ;

- les techniques relatives aux opérations de transformation, de conditionnement et de stockage de l'huile d'olive.

\section{1 Échantillonnage}

Les échantillons d'huile d'olive analysés et qui ont fait objet du présent travail ont été collectés durant les deux campagnes 2006/2007 et 2009/2010, à partir d'unités de trituration situées dans la région du Sahel et du centre tunisien (les gouvernorats de Monastir, Sousse, Mahdia et Sidi Bouzid).

L'échantillonnage concerne des huile d'olive produites par 60 huileries équipées en différents systèmes d'extraction : des huileries en phases truelle, d'autres en phases duelle et des huileries traditionnelles équipées en systèmes de presses ou de super-presses.

\subsection{Les paramètres physico-chimiques de l'huile}

Les paramètres physico-chimiques comme l'acidité libre, l'indice de peroxyde et les coefficients d'extinction spécifique à 232, 270, 274 nm ont été évalué suivant la méthode officielle décrite dans le règlement $\mathrm{CCE} / 2568 / 91$ de la Commission des Communautés Européennes (CCE, 1991).

\subsection{Analyse sensorielle : panel de jury entraîné}

L'analyse sensorielle de l'huile d'olive vierge. Les membres du panel sont entrainés à reconnaitre les différentes flaveurs caractéristiques de l'huile d'olive et à mesurer leurs intensités en se référant à la norme du COI «Méthodologie générale pour l'évaluation sensorielle de l'huile d'olive vierge » et la norme COI «Guide pour la sélection, l'entraînement et le contrôle des dégustateurs qualifiés de l'huile d'olive vierge » (COI, 2006).

\subsection{Analyse satatistique}

Les analyses statistiques des données sont réalisées à l'aide du logiciel SPSS version 11.0 (SPSS, Chicago, IL, USA).

\section{Resultats et discussion}

\subsection{La récolte des olives}

La récolte est une opération importante de la culture de l'olivier et, par conséquent, elle doit être contrôlée de près étant donné ses répercussions sur le coût de la production et la qualité de l'huile d'olive. Cette dernière est affectée aussi bien par les modalités de récolte (système, durée) que par l'époque à laquelle intervient celle-ci (ONUDI, 2007).

\subsection{1 État de la matière première}

Les résultats de l'enquête en ce qui concerne l'état de la matière première sont décrites dans la Figure 1.

La plupart des olives triturées sont des olives fraîches (59\%), car les agriculteurs ramènent leurs olives à l'huilerie dans des délais brèves et parfois le jour même de la récolte. La Figure 1 révèle aussi qu'une minorité des agriculteurs $(5 \%)$ procèdent au mélange des olives de bonne et de mauvaise qualité (en termes de fraicheur). Les olives sont en général d'aspect sanitaire bon (47\%), relativement humides $(51 \%)$ et n'ayant pas subit la fermentation.

La croissance des olives passent par trois phases : une première phase rapide, suivie d'une deuxième phase au cours de laquelle la croissance est lente, entre août et septembre, 

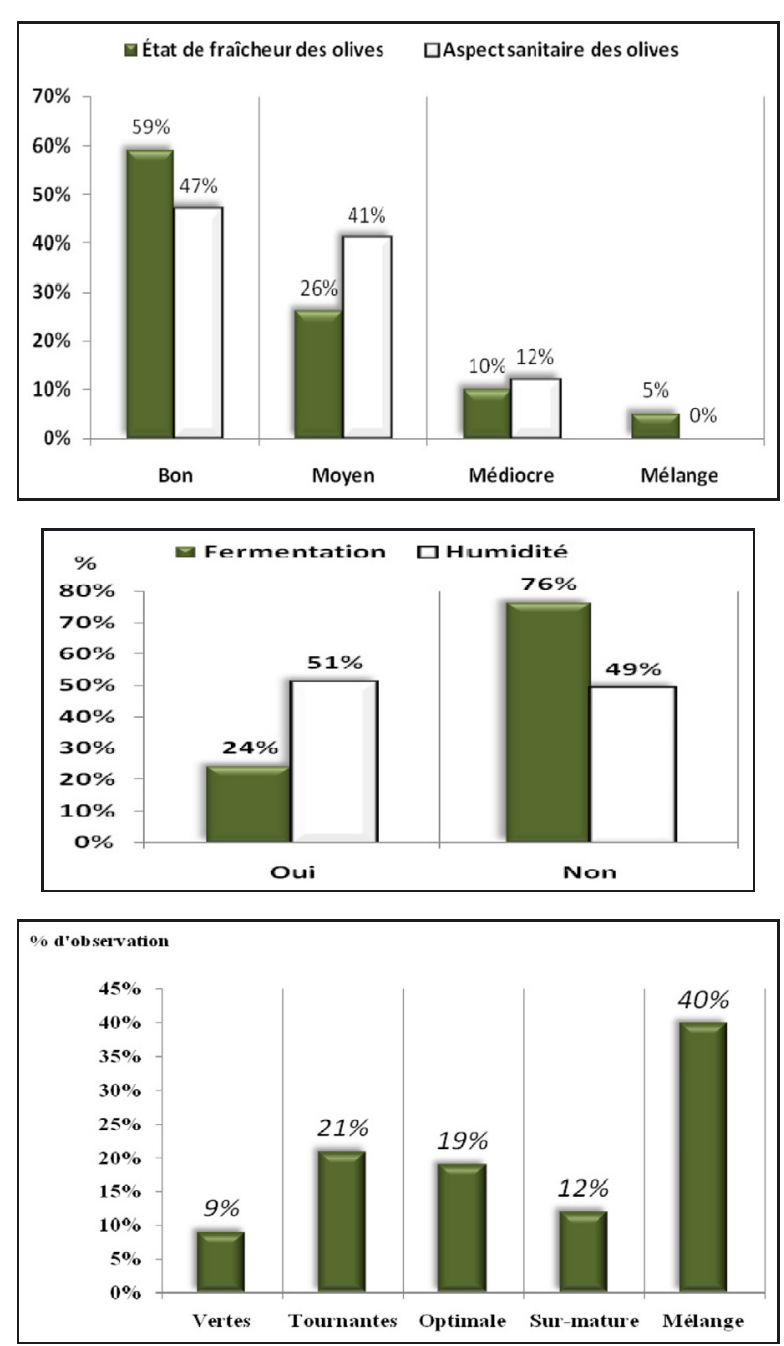

Fig. 1. Description de l'état des olives avant leur trituration $(n=88)$.

et une troisième phase rapide en automne au cours de laquelle le fruit change de couleur (Chimi, 2001). Le degré de maturité des olives revêt un intérêt primordial pour l'obtention d'huiles d'olive de bonne qualité. Pratiquement, une huile d'olive vierge est considérée comme étant de bonne qualité lorsque la cueillette des fruits est effectuée au stade optimal de récolte des olives qui est représenté par le stade de maturité semi-noir (ONUDI, 2007). De l'enquête, il ressort que $31 \%$ des olives sont triturées au stade noir, $30 \%$ aux stades verts à tournantes tandisque $40 \%$ des unités triturent des mélanges d'olives de stades de maturité différents (vertes et noires), ce pourcentage s'approche de celui publié par Chimi (2001) pour une enquête menée au Maroc (45\%).

\subsubsection{Systèmes de récolte}

Généralement, la récolte des olives est traditionnelle; $84,4 \%$ des agriculteurs utilisent la récolte manuelle alors que 9,1\% seulement pratiquent le gaulage (Fig. 2) par opposition au Maroc où le gaulage est le système principal de récolte $(90 \%)$ et les $10 \%$ restant concernent la récolte naturelle : les agriculteurs ramassent les olives manuellement lorsqu'elles sont tombées (Chimi, 2001).

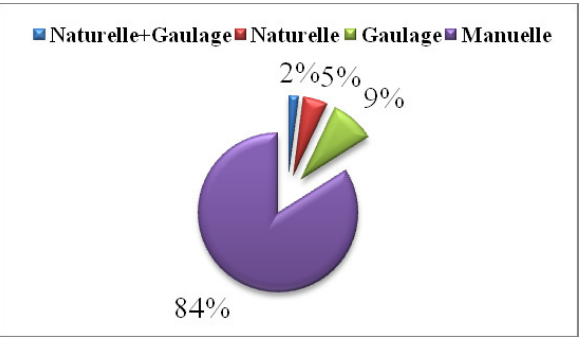

Fig. 2. Différents types de récolte utilisés par les agriculteurs enquêtés $(n=77)$.

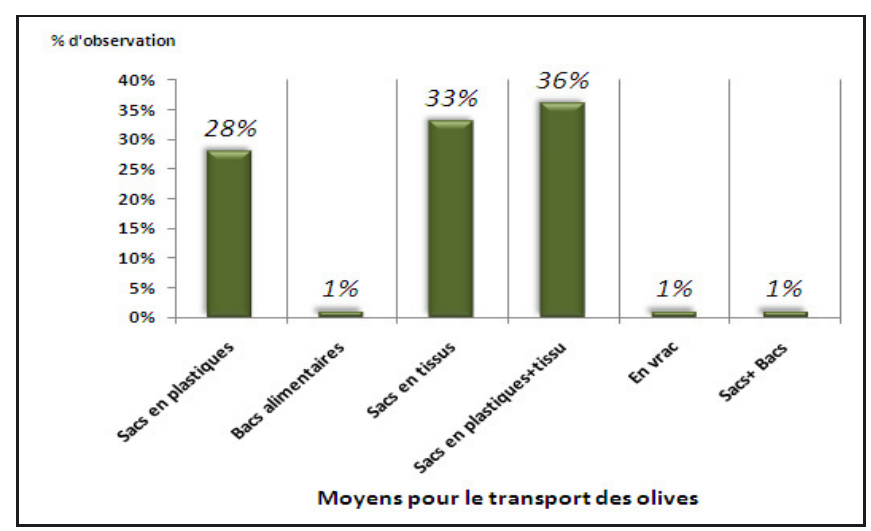

Fig. 3. Transport des olives aux unités de trituration $(n=89)$.

En fait, la qualité du produit final dépend du traitement. Il faut donc éviter de choquer ou de blesser les fruits au cours du ramassage (gaulage) : tout choc ou blessure est le lien de développement de micro-organismes avec les conséquences de dégradation de l'huile qu'ils peuvent entraîner (François, 1974). La cueillette à la main ou à l'aide d'un peigne, directement sur l'olivier, est une activité consommatrice de main d'œuvre. Mais elle présente l'avantage de ne pas endommager les branches et les rameaux.

\subsection{Transport des olives}

Dans le souci de conserver les caractéristiques de qualité que les olives possèdent au moment de la récolte sur l'arbre, il s'avère nécessaire de les acheminer immédiatement vers le moulin (ONUDI, 2007). Selon les données de l'enquête, on peut remarquer que dans la plupart des cas, le transport des olives se fait d'une manière relativement non conforme (53\%). Les sacs en tissu (en jute) ainsi que les sacs en plastiques sont pratiquement le moyen principal $(97 \%)$ utilisés pour l'acheminement des olives au moulin (Fig. 3).

En revanche, le moyen le plus approprié pour le transport des olives est représenté par les caisses à claire voie en matière plastique permettant la circulation de l'air et évitant des réchauffements préjudiciables causés par l'activité catabolique des fruits (Garcia et Streif, 1991). Ces caisses limitent l'épaisseur de la couche d'olives et réduisent donc le danger d'écrasement, tout en représentant un moyen idéal pour le stockage en attendant le pressage (García et Yousfi, 2006). En revanche, le transport des olives dans des sacs en jute est peu rationnel, car cette modalité provoque inévitablement des lésions aux drupes, surtout si elles sont très mûres. Elles sont à l'origine 


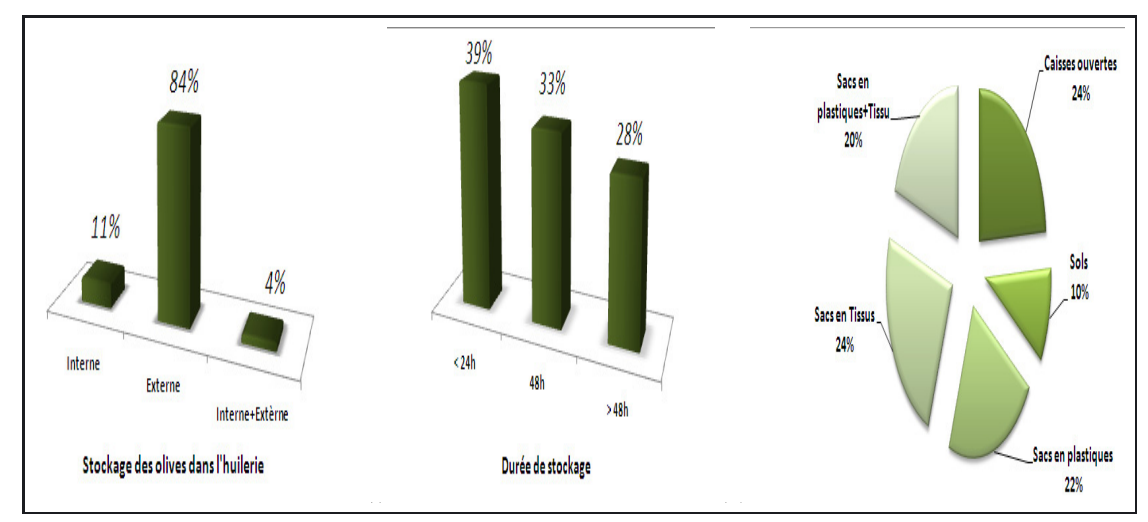

Fig. 4. Maîtrise de l'entreposage des olives dans les huileries enquêtées $(n=41)$.

du déclenchement de processus biologiques d'altération de la qualité de l'huile (ONUDI, 2007).

\subsection{Stockage des olives dans l'huilerie}

Les olives, avant d'être trituré, subissent un stockage dans l'huilerie qui est le plus souvent externe (84\% des cas). La trituration des olives est pratiquée dans des délais ne dépassant pas les 48 h qui suivent l'acheminement des olives dans $72 \%$ des cas. Les olives peuvent chômer pendant une durée dépassant parfois les $48 \mathrm{~h}$ surtout pour les unités traditionnelles (presses) et les unités industrielles équipées en super-presses avec un pourcentage de $28 \%$. En effet, Le caractère saisonnier de la production oléicole, les problèmes de transport et les autres contraintes liées aux structures de la filière oléicole, ne permettent généralement pas d'adapter le rythme de réception aux capacités des unités de trituration; d'où le nécessaire recours au stockage (Garcia et Streif, 1991; Gutierrez et al., 1992). Le stockage s'impose quand la cadence de réception est supérieure à la capacité de trituration, mais également dans le cas inverse; dans ce cas le stockage a pour but la constitution d'une quantité d'olive suffisante pour alimenter les machines pendant une durée minimale économiquement acceptable. Le stockage est donc un mal nécessaire et constitue dans la majorité des cas la principale cause de la détérioration de la qualité de l'huile extraite (ONUDI, 2007) (Olias et Garcia, 1997).

Concernant les conteneurs adoptés pour le stockage des olives avant leur trituration, on remarque que la plupart des huileries enquêtées ne consacrent pas de conteneurs spéciaux pour le stockage et que dans la plupart des cas les olives sont gardées dans les sacs avec lesquels elles ont été acheminées $(65,9 \%)$; ce pourcentage est de $6 \%$ seulement au Maroc (Chimi, 2001). Ce sont surtout des unités de trituration équipées en chaîne continue à 2 phases qui consacrent des caisses ouvertes $(24,4 \%)$. Le stockage des olives sur le sol semble être très rare $(9,8 \%)$ et rencontré essentiellement dans les unités utilisant des systèmes de pression (Fig. 4). Le stockage des olives en vrac est le principal mode de stockage des olives dans les huileries marocaines (Chimi, 2001; García et Yousfi, 2006).

Après leur récolte et avant leur broyage, les olives ne doivent pas être stockées pendant une durée supérieure à $48 \mathrm{~h}$. Elles doivent être conservées dans des caisses en plastique perforées qui permettent d'éviter des couches d'olives trop
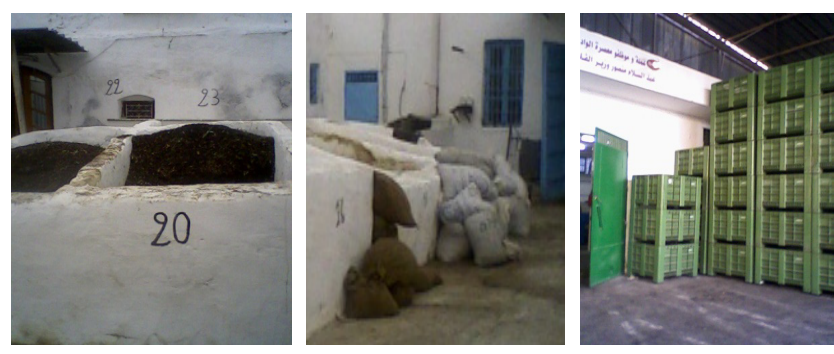

Fig. 5. Différents modes de stockage des olives dans les huileries Tunisiennes.

épaisses, ce qui pourrait provoquer l'écrasement des fruits avec les répercussions négatives inévitables que cela suppose sur la qualité de l'huile. Il est recommandé que ces caisses soient de format large $\left(100 \times 100 \times 120 \mathrm{~cm}^{3}\right)$ pour une durée de stockage $\leqslant 48 \mathrm{~h}$ et de petit format pour un stockage prolongé $\left(50 \times 30 \times 40 \mathrm{~cm}^{3}\right)$ (Tous et al., 2005). D'ailleurs, l'emploi des caisses en plastique perforées s'avère pertinent et utile car il permet l'aération des olives (García et Yousfi, 2006). Il convient également d'éviter le stockage des olives en gros tas en contact direct avec le sol à cause de l'entassement que subissent les fruits, sinon il doit être réalisé de façon à ce que le rapport superficie/volume soit le plus faible possible (couches minces de 20 à $25 \mathrm{~cm}$ ). L'emploi de sacs, en jute ou en plastique, pour la conservation des olives en attendant le broyage est totalement proscrit (ONUDI, 2007) (Fig. 5).

\subsection{Maîtrise des locaux}

Selon les données de l'enquête, on peut remarquer que la plupart des huileries mettent en place un plan de maintenance des locaux $(63 \%)$ et ceci au début et en fin de campagne seulement alors qu'une minorité des huileries adopte un plan de maintenance durant la campagne oléicole. Les opérations de nettoyage/désinfection sont rarement pratiquées (39\% des huileries) (Fig. 6).

Le responsable du moulin doit établir un plan de maintenance des locaux, préférentiellement préventif, appliqué entre deux campagnes de production : réfection des peintures et des sols, changement de carreaux fêlés, décollés ou cassés, entretien des ouvertures, des systèmes de ventilation, des évacuations, des sanitaires, etc. (COI, 2003). 

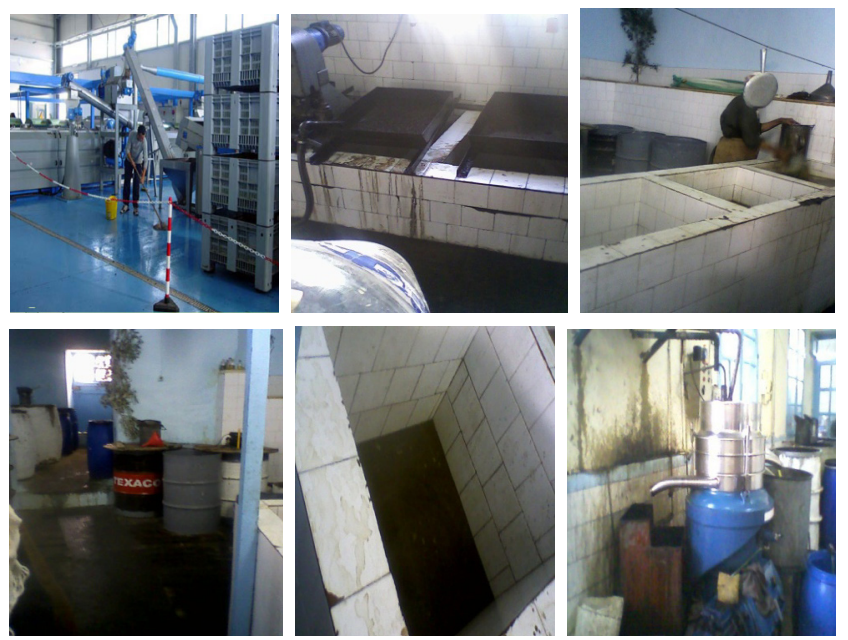

Fig. 6. Hygiène des locaux au sein des huileries enquêtées.

Les opérations de nettoyage/désinfection participent à la maîtrise préventive de la contamination des produits (olives et huiles). En fait, un programme permanent de nettoyage/désinfection doit être appliquer au sein du moulin en cours de campagne ainsi que entre deux campagnes de trituration. Ce programme permet donc de s'assurer que toutes les parties de l'unité sont traitées de façon appropriée (AFIDOL, 2003). Les locaux doivent être entretenus par le personnel qui y travaille et supervisé par le chef d'équipe désigné. La supervision concerne l'indication des moments et des méthodes de nettoyage à appliquer (USAID/Maroc, 2006).

\subsection{Maîtrise du matériel}

D'après le Tableau 3, on peut remarquer qu'il y a mise en place de plan de maintenance et de nettoyage/désinfection pour l'ensemble des équipements surtout pour les unités de trituration à deux phases.

Le matériel en contact direct avec les olives et les huiles doit être conçu de manière à permettre un bon contrôle et un nettoyage quotidien (Afidol, 2003).

En ce qui concerne les produits de nettoyage et de désinfection, la majorité des huileries $(71 \%)$ utilisent des produits homologués (cristaux). Cela n'empêche qu'un nombre non négligeable d'huileries (29\%) utilisent des produits non homologués tels que l'Omo, la soude et parfois de l'eau chaude uniquement. Les produits de nettoyage-désinfection doivent répondre à la réglementation liée aux aliments (décret No. 73/138 du 12/02/73 modifié et son arrêté d'application du 08/09/99 (J.O. du 27/11/99); les désinfectants doivent en outre avoir fait l'objet d'une autorisation de mise sur le marché comme les fongicides, bactéricides ou virucides (AFIDOL, 2003).

\subsection{Maîtrise de la main d'œuvre}

L'hygiène corporelle n'est pas respectée dans 58,5\% des huileries enquêtées et la tenue vestimentaire du personnel n'est pas appropriée (41,5\%). Généralement, et dans 73,5\% des cas, la formation du personnel en matière de nettoyage/désinfection, maintenance, etc. est absente. De plus,
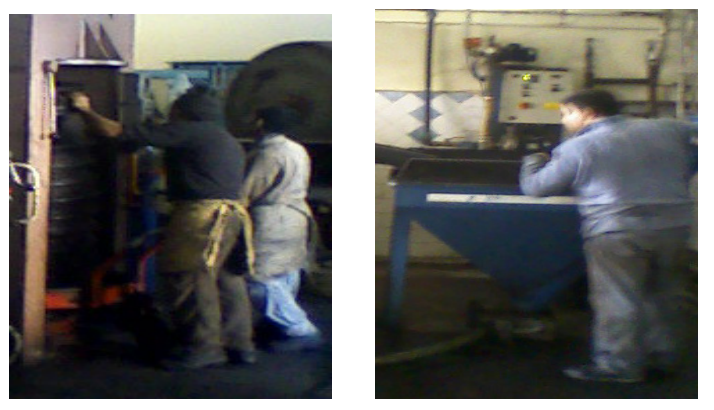

Fig. 7. Tenue vestimentaire inaproprié du personnel d'huileries.

on note l'absence d'affiches de réclamation pour le personnel dans $70,7 \%$ des cas.

En outre, les personnes qui sont en contact direct avec les fruits doivent être soumis à un règlement bien établi et affiché à l'intérieur de l'unité. Des mesures strictes doivent être prises pour l'application effective des mesures de propreté. Ces mesures doivent englober tous les points qui peuvent affecter la qualité des olives ainsi que celle des huiles d'olive (USAID/Maroc, 2006) (Fig. 7). En effet le personnel doit porter des vêtements de travail (blouses ou combinaisons), entretenus propres, et se laver les mains à la sortie des toilettes avant de regagner le poste de travail. Le tabac doit être interdit et des distributeurs d'eau potable doivent être mis à la disposition des ouvriers (ONUDI, 2007).

\subsection{Maîtrise des méthodes : étapes de trituration}

\subsubsection{Lavage et effeuillage des olives}

Avant d'initier le cycle de mise en œuvre proprement dit, les olives destinées à la production d'huile de qualité font l'objet des opérations d'effeuillage et de lavage. Ces opérations sont effectuées en général par une seule machine conçue à cet effet, qui réalise les deux opérations en séquence. L'effeuillage et le lavage sont importants non seulement pour des raisons d'hygiène et de santé mais également pour des raisons techniques, mécaniques et de qualité de l'huile (Di Giovacchino et al., 2002).

À travers les résultats de l'enquête, on a pu mettre en évidence que les opérations de lavage et d'effeuillage des olives ne sont pratiquées que dans le cas de quelques huileries équipées en presses et en super-presse correspondant à un pourcentage de $15 \%$ seulement (Fig. 8). Ce pourcentage est relativement faible par rapport à celui rapporté dans l'étude menée au Maroc par Chimi (2001) qui a montré que le lavage des olives n'est pas pratiqué dans $61 \%$ des huileries Marocaines. L'eau utilisée peut être de l'eau de robinet, eau de puits traitée ou eau de forage (Fig. 8).

\subsubsection{Durée du broyage}

La plus grande partie des échantillons étudiés (52,8\%) a subit un broyage dont la durée est comprise entre 20 et $30 \mathrm{~min}$ conformément à la norme du COI (Fig. 9). Dans le tiers des unités traditionnelles $(33,3 \%)$, la durée du broyage est supérieure à $30 \mathrm{~min}$; alors que $57 \%$ des unités traditionnelles marocaine pratiquent une mouture prolongée (>90 min) 


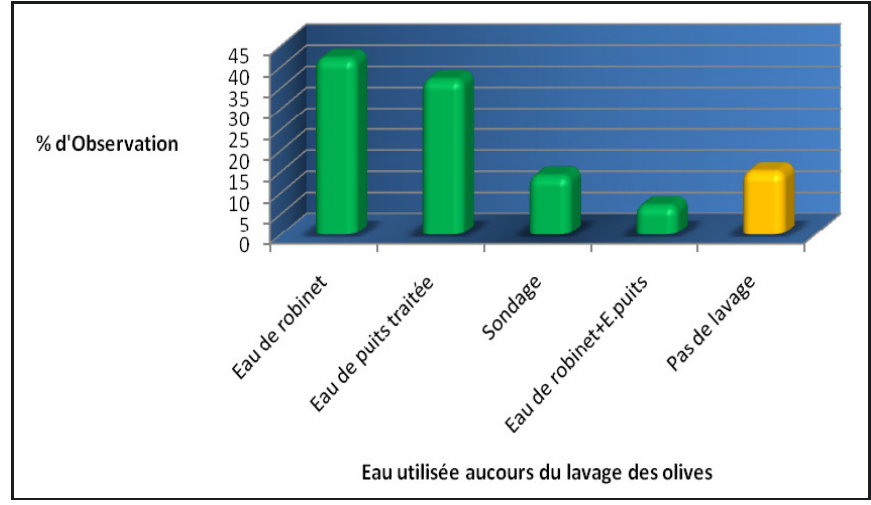

Fig. 8. Description statistique de l'étape de lavage des olives $(n=86)$.

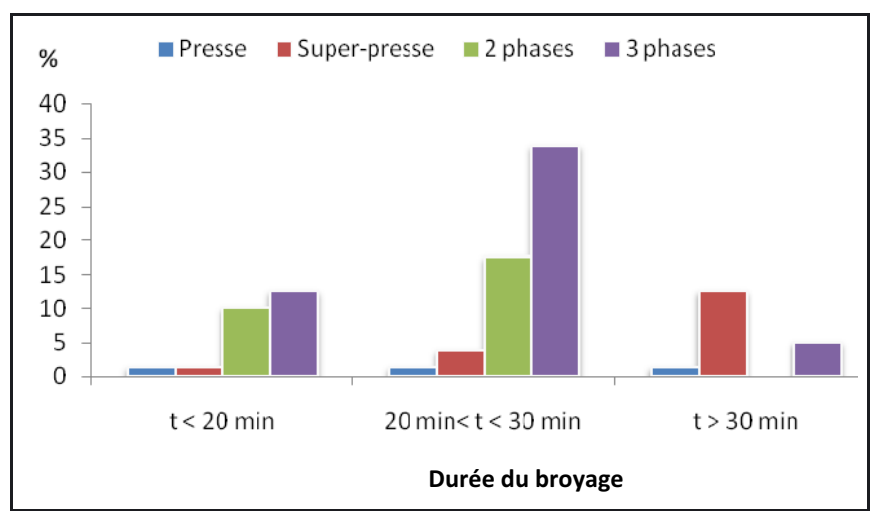

Fig. 9. Durée du broyage $(n=80)$.

(Chimi, 2001). Pour les unités industrielles tunisiennes, la durée est plus courte (57\% entre 20 et $30 \mathrm{~min}$ ).

Selon la norme du Conseil Oléicole International, la durée de broyage est variable en fonction des particularités des olives et ne doit pas dépasser 15 à 30 min pour les systèmes de pression et 20 à 30 min pour les systèmes de centrifugation (COI, 2006).

\subsubsection{Le malaxage}

L'étape de malaxage se déroule dans la plupart des huileries enquêtées pendant une durée ne dépassant pas les $60 \mathrm{~min}$, ainsi la durée du malaxage est maîtrisée. En revanche, la température de malaxage pour plus que la moitié des échantillons (52\%) dépasse la valeur fixée par la norme du COI (COI, 2003) (Fig. 10).

La température du malaxage doit être bien contrôlée, grâce au thermostat dont l'appareil est doté, pour une préparation adéquate de la pâte d'olives et pour éviter d'éventuelles conséquences négatives sur l'huile produite. Quelque soit le système adopté dans l'huilerie, la température de malaxage ne devrait pas être supérieure à $25^{\circ} \mathrm{C}$. Cette valeur assure, selon les caractéristiques et la qualité des olives, de bons rendements à l'extraction ainsi qu'une bonne qualité de l'huile (COI, 2006) (Fig. 10).

\subsubsection{Quantité d'eau ajoutée}

La quantité d'eau ajoutée pendant l'extraction atteint souvent $20 \mathrm{~L} / 100 \mathrm{~kg}$ d'olives dans $38 \%$ des cas étudiés essen- tiellement pour les systèmes de centrifugation à trois phases. Pour les unités équipées en presses, super-presses ou en deux phases, l'ajout de l'eau n'est pas souvent nécessaire sauf si les olives ne sont pas humides, cela dépend donc de la campagne oléicole (Fig. 11).

Le décanteur centrifuge à trois phases nécessite l'addition de 40-60 1/100 kg d'olives, en revanche le système de centrifugation à deux phases ainsi que le système de pression ne nécessitent pas l'adjonction de l'eau (Di Giovacchino et al., 2002).

\subsubsection{Stockage de l'huile d'olive}

Une fois produite dans le séparateur centrifuge vertical, l'huile d'olive vierge est encore plus ou moins trouble, car elle contient en émulsion de petites quantités d'eau de végétation et des micro-fragments végétaux qui tendent avec le temps à se sédimenter. Avant d'être conditionnée, cette huile trouble doit être emmagasinée par lots homogènes dans des réservoirs en acier inoxydable qui sont installés dans un environnement de stockage adapté et à une température qui varie entre 13 et $18^{\circ} \mathrm{C}$.

Différents types de réservoirs sont utilisés au niveau des huileries tunisiennes pour stocker l'huile d'olive. En effet, la grande majorité des huileries utilisent, pour le stockage de l'huile d'olive, les bassines en faïence en premier lieu, ensuite viennent les barils en plastique non alimentaire, les piles et à moindre degré les citernes en inox pour les huileries à haute capacité de trituration (Fig. 12).

\subsubsection{Traçabilité et système HACCP}

La plupart des huileries présente un système de traçabilité simple : il est remarquable que la plupart des responsables des moulins n'ont pas une grande idée sur la traçabilité (qui est utilisée uniquement dans $57 \%$ des huileries enquêtées), ni sur le système HACCP qui est presque absent (21\% des huileries mettent en place un système HACCP). La traçabilité et l'application des principes du système HACCP à la fabrication d'huile d'olive permettront aux unités d'établir un système de contrôle, visant à maîtriser l'ensemble des facteurs intervenant au cours de la conduite technologique, en vue d'obtenir une huile d'olive saine et de qualité (Afidol, 2003).

\subsection{Caractéristiques physicochimiques d'huile d'olive}

Le Tableau 1 présente les caractéristiques physicochimiques des différents échantillons d'huile d'olive prélevés auprès des unités de trituration traditionnelles et industrielles implantées dans différentes régions oléicoles de la zone du Sahel et centre tunisien. Une partie de ces huiles d'olives possèdent des caractéristiques physicochimiques d'huile d'olive vierge à extra vierge et sans défaut majeur. Les résultats des analyses de l'acidité, de l'indice de peroxyde et l'analyse spectrophotométrique des échantillons d'huiles d'olive provenant des coopératives concernées ont été comparés à la norme commerciale du COI. Cette comparaison a révélé que tous les échantillons sont conformes à la norme du COI : l'acidité et l'indice de peroxyde respectent les valeurs données par le COI. 


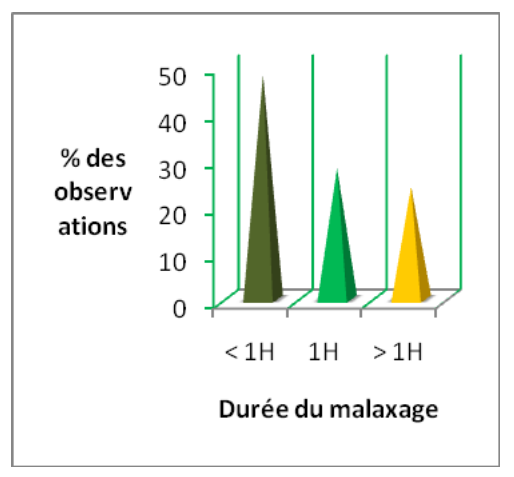

Fig. 10. Description de l'opération de malaxage $(n=86)$.

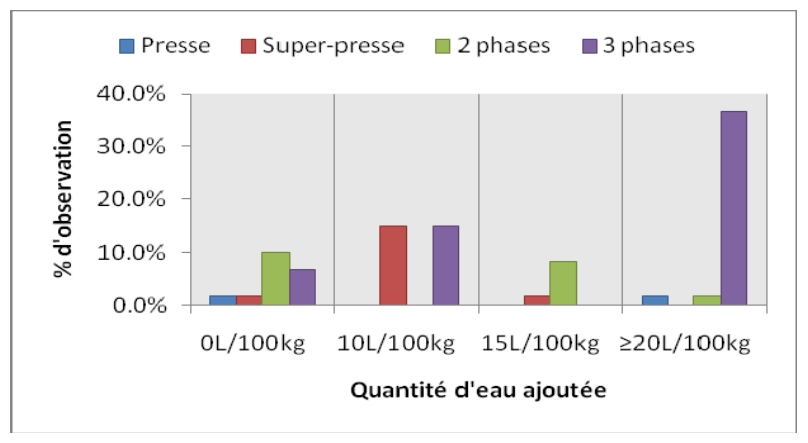

Fig. 11. Quantité d'eau ajoutée au cours de la trituration en fonction des différents systèmes d'extraction $(n=64)$.

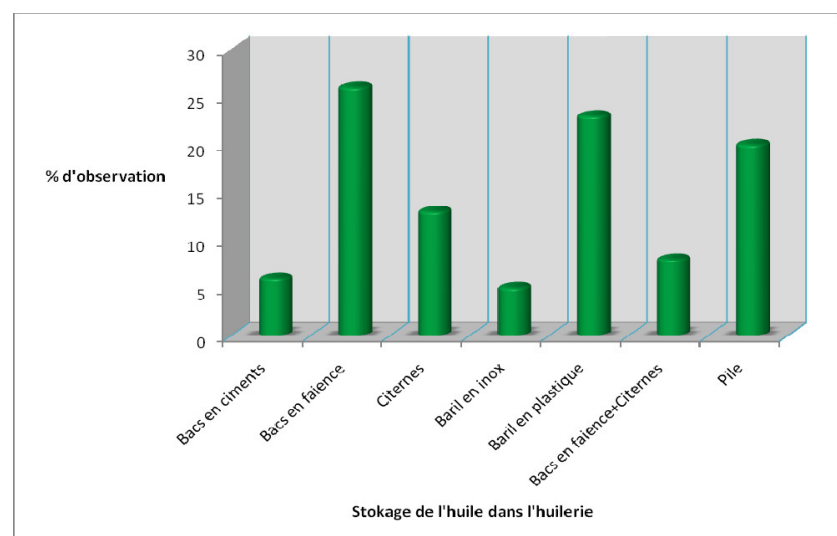

Fig. 12. Conteneurs utilisés pour le stockage de l'huile dans les huileries enquêtées $(n=88)$.

Les extinctions spécifiques dans l'ultraviolet (K232, K270) et le $\Delta K$ présentent des valeurs généralement faibles pour la majorité des échantillons et restent en dessous de la limite autorisée par la norme. Certains échantillons, en revanche, ont présenté des valeurs se situant à la valeur limite supérieure audessus de laquelle ces huiles d'olive risqueraient d'être déclassées dans la catégorie d'huile d'olive courante, voire lampante.

D'après le Tableau 1, on peut remarquer une amélioration de la qualité d'huile d'olive en passant du système de pression aux systèmes à centrifugation par augmentation du pourcentage des huiles propres à la consommation en l'état (vierges, extra vierges et courantes). Les résultats de la physicochimie montrent que la plupart des huiles produites sont de la caté-

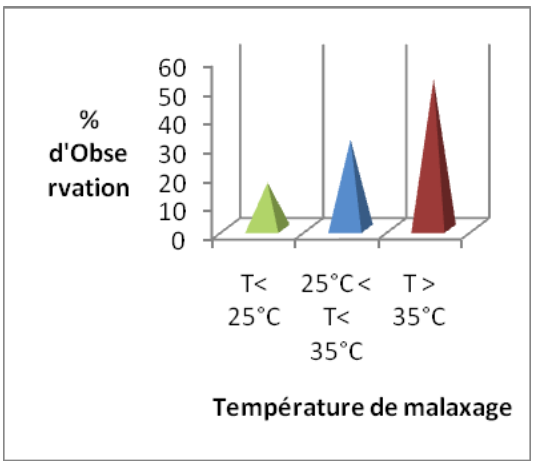

gorie extra vierge voire vierges alors que l'analyse sensorielle révèle que la plupart des échantillons sont des huiles lampantes (78 \%). Les défauts majeurs retrouvés sont le chaumé (flaveur caractéristique de l'huile tirée d'olives entassées ou stockées dans des conditions telles qu'elles se trouvent dans un état avancé de fermentation anaérobie), le rance (flaveur des huiles ayant subi un processus d'oxydation intense) et le moisi (flaveur caractéristique de l'huile obtenue d'olives attaquées par des moisissures et des levures suite à un stockage des fruits pendant plusieurs jours dans l'humidité ou de l'huile obtenue d'olives ramassées avec de la terre).

En effet, les facteurs à incriminer et qui peuvent expliquer la mauvaise qualité des huiles produites par pression, concernent essentiellement la récolte des olives, la durée de chômage prolongée des olives dans l'huilerie, les opérations de triage, de lavage et d'effeuillage qui sont rarement pratiquées, le broyage inadéquat dont la durée dépasse parfois $1 \mathrm{~h}$, le problème de nettoyage des scourtins qui n'est pas toujours réalisé et le temps de séparation de l'huile des autres phases qui reste prolongé. Ces résultats sont en accord avec celles publiées par Chimi (2001) qui a travaillé sur la variété picholine marocaine.

En revanche, l'amélioration de la qualité des huiles produites par centrifugation est due à (Gharbi et al., 2014) :

- la récolte des olives qui se fait entre le stade semi-noir et noir ce qui correspond au stade optimal de récolte des olives, paramètre déterminant sur la qualité de l'huile d'olive;

- le chômage des olives qui est presque inexistant;

- les opérations de lavage et de broyage qui sont pratiqués;

- le broyage adéquat (temps assez court) suite à l'utilisation des broyeurs à marteaux ;

- l'étape de séparation de l'huile des autres phases est rapide et se fait par centrifugation.

\section{Conclusion}

La qualité de l'huile d'olive est le résultat d'approches variées et complexes : elle peut être perçue comme une chaine débutant au moment de la plantation d'une variété donnée d'olivier, en passant par les conditions de culture, de transformation, de stockage et de distribution de l'huile jusqu'à son arrivée au niveau consommateur. L'application des BPH et du HACCP permettra de répondre aux exigences des marchés en termes de qualité sanitaire et de traçabilité. 
Tableau 1. Caractéristiques physico-chimiques d'huile d'olive produites dans les unités traditionnelles et industrielles.

\begin{tabular}{|c|c|c|c|c|c|c|c|}
\hline \multirow{2}{*}{$\begin{array}{l}\text { Indice de } \\
\text { qualité }\end{array}$} & \multirow{2}{*}{ Limite de l'indice } & \multirow{2}{*}{ Type huile d'olive } & \multicolumn{5}{|c|}{ Résultats d'analyse (\%) } \\
\hline & & & Presse & Super-presse & 2 phases & 3 phases & Total \\
\hline \multirow{4}{*}{$\begin{array}{l}\text { Acidité libre } \\
\quad(n=41)\end{array}$} & $\leqslant 0,8$ & Extra vierge & $n d$ & 0 & 17,1 & 22,0 & 39,0 \\
\hline & $\leqslant 2,0$ & Vierge & nd & 7,3 & 2,4 & 29,3 & 39,0 \\
\hline & $\leqslant 3,3$ & Courante & $n d$ & 2,4 & 2,4 & 9,8 & 14,6 \\
\hline & $>3,0$ & Lampante & $n d$ & 2,4 & 0 & 4,9 & 7,3 \\
\hline \multirow{2}{*}{$\begin{array}{c}\text { Indice de Peroxyde } \\
(n=75)\end{array}$} & $\leqslant 20$ & extra -vierge-courante & 2,7 & 14,7 & 28,0 & 54,7 & 100 \\
\hline & $>20$ & Lampante & 0 & 0 & 0 & 0 & 0 \\
\hline \multirow{4}{*}{$\begin{array}{c}\mathrm{K} 270 \\
(n=41)\end{array}$} & $\leqslant 0,22$ & Extra vierge & $n d$ & 9,8 & 19,5 & 46,3 & 75,6 \\
\hline & $\leqslant 0,25$ & Vierge & $n d$ & 0 & 0 & 4,9 & 4,9 \\
\hline & $\leqslant 0,30$ & Courante & $n d$ & 2,4 & 2,4 & 9,8 & 14,6 \\
\hline & $>0,30$ & Lampante & $n d$ & 0 & 0 & 4,9 & 4,9 \\
\hline \multirow{3}{*}{$\begin{array}{c}\mathrm{K} 232 \\
(n=41)\end{array}$} & $\leqslant 2,50$ & Extra & $\overline{n d}$ & 9,8 & 19,5 & 61,0 & 90,2 \\
\hline & $\leqslant 2,60$ & Vierge-courante & $n d$ & 2,4 & 0 & 2,4 & 4,9 \\
\hline & $<3,70$ & lampante & $n d$ & 0 & 2,4 & 2,4 & 4,9 \\
\hline \multirow{2}{*}{$\begin{array}{c}\Delta k \\
(n=41)\end{array}$} & $\leqslant 0,01$ & extra -vierge-courante & nd & $\overline{9,8}$ & 19,5 & 58,5 & 87,8 \\
\hline & $>0,01$ & Lampante & $n d$ & 2,4 & 2,4 & 7,3 & 12,2 \\
\hline \multirow{4}{*}{$\begin{array}{l}\text { Analyse sensorielle } \\
\qquad(n=41)\end{array}$} & & Extra vierge & $n d$ & 0 & 2,4 & 2,4 & 4,9 \\
\hline & & Vierge & $n d$ & 0 & 7,3 & 7,3 & 14,6 \\
\hline & & Courante & $n d$ & 0 & 0 & 2,4 & 2,4 \\
\hline & & Lampante & nd & 12,2 & 12,2 & 53,7 & 78,0 \\
\hline \multirow{6}{*}{$\begin{array}{c}\text { Défauts } \\
\text { sensoriels } \\
(n=41)\end{array}$} & & Aucun & nd & 0 & 2,4 & 2,4 & 4,9 \\
\hline & & Chaumé & $n d$ & 0 & 2,4 & 4,9 & 7,3 \\
\hline & & Chaumé + Rance & nd & 12,2 & 14,6 & 53,7 & 80,5 \\
\hline & & Chaumé+Rance+moisi & nd & 0 & 0 & 2,4 & 2,4 \\
\hline & & Moisi + Chaumé & $n d$ & 0 & 2,4 & 0 & 2,4 \\
\hline & & Rance + Moisi & $n d$ & 0 & 0 & 2,4 & 2,4 \\
\hline
\end{tabular}

$n d:$ non déterminée.

\section{Références}

AFIDOL. 2003. Association Française Inter-professionnelle de l'Olive. Guide de Bonnes Pratiques d'Hygiène pour la fabrication d'Huile d'Olive Vierge. Version indice 7.

CCE. 1991. Commission regulation (CCE) no. 2568/91 of 11 July 1991 on the characteristics of olive oil and olive-residue oil and on the relevant methods of analysis. Official Journal L 248: 0001-0083.

Chimi H. 2001. Qualité des huiles d'olive au Maroc-Enquête nationale et analyses au laboratoire. Transfert de technologie en agriculture. MADREF/DERD, 97.

COI. 2006. Conseil oléicole international. Guide de gestion de la qualité de l'industrie de l'huile d'olive : les moulins. T.33/Doc. No. 2-4.

Di Giovacchino L, Costantini N, Ferrante ML, Serraiocco A. 2002. Influence of malaxation time of olive paste on the technological results and qualitative characteristics of virgin olive oil obtained by a centrifugal decanter at water saving. Grasas Aceites.

François R. 1974. «Industrie des corps gras, biochimie, extraction, raffinage et réglementation ». Ed. tech et doc, Genève.

García JM, Yousfi K. 2006. The postharvest of mill olives. Grasas y Aceites 57: 16-24.

Garcia JM, Streif J. 1991. The effect of controlled atmosphere storage on fruit quality of "Gordal" olives. Gartenbauwissenschaft 56: 233-238.
Gharbi I, Issaoui M, Hammami M. 2014. La filière huile d'olive en Tunisie. OCL 21: D202.

Gutierrez F, Perdiguero S, Garcia JM, Castellano JM. 1992. Quality of oils from olives stored under controlled atmosphere. J. Am. Oil Chem. Soc. 69: 1215-1218.

Hannachi H, Msallem M, Ben Elhadj S, El Gazzah M. 2007. Influence du site géographique sur les potentialités agronomiques et technologiques de l'olivier (Olea europaea L.) en Tunisie. Compte Rendus Biologies 330: 135-142.

Karray B, Msallam M, Ksantini M, Mahjoub Boujnah D, Grati Kamoun N. 2009. Institut de l'Olivier - Tunisie Programmes et acquis de recherches pour la rénovation de la filière huile d'olive et l'amélioration de ses performances.

Olias JM, Garcia JM. Olive. In : Mitra SK, (Ed.), Postharvest Physiology and Storage of Tropical and Subtropical Fruits. CAB International, Wallingford, UK, 1997, pp. 229-243.

ONUDI. 2007. Organisation des Nations Unies pour le developpement Industriel. Guide du producteur de l'huile d'olive.

Tous J, Villamil J, Hermoso JF, Alber A. 2005. El olivo en Uruguay. Olivae 103: 56-61.

USAID/Maroc. 2006. Agence Américaine pour le Developpement International. Projet des huiles d'olives vierges Sais-Meknes. Manuel des bonnes pratiques d'hygiène (BPH) des huiles d'olives vierges. Agriculture $\mathcal{E}$ Agrobusiness intégrés. 